\title{
Effects of specimen and grain size on electrically-induced softening behavior in uniaxial micro-tension of AZ31 magnesium alloy: experiment and modeling
}

\author{
Xinwei Wang ${ }^{a, b, c}$, Jie Xu ${ }^{\text {b,c* }}$, Debin Shan ${ }^{b, c}$, Bin Guo $^{\text {b,c }}$, Jian Cao $^{a^{*}}$ \\ ${ }^{\text {a }}$ Department of Mechanical Engineering, Northwestern University, 2145 Sheridan Rd, Evanston, IL \\ 60208, USA
}

${ }^{\mathrm{b}}$ Key Laboratory of Micro-Systems and Micro-Structures Manufacturing of Ministry of Education, Harbin Institute of Technology, Harbin 150080, China

${ }^{c}$ School of Materials Science and Engineering, Harbin Institute of Technology, Harbin 150001, China

\begin{abstract}
Various electrically-assisted (EA) plastic forming technologies have been developed for difficult-to-form materials such as magnesium alloys in recent years. However, very few studies have been conducted on EA micro-forming, especially determining the size effect on electrically-induced softening behavior. In this study, uniaxial micro-tension tests at various current densities were conducted to investigate the effects of grain size and specimen size on the electrically-induced softening behavior of magnesium alloy AZ31 specimens. It was found that the electrically-induced softening parameter (i.e., the ratio of the tensile strength in EA to that in non-EA test with the higher softening at the smaller value) followed an inverse-S-shaped function of current density. A relatively lower current density would be sufficient for larger sample sizes and smaller grain sizes to achieve a higher softening effect, indicating that grain number may be an important factor influencing electrically-induced softening. These size effects on electrically-induced softening were used to modify a semi-empirical softening function of current density, which could effectively predict the electrically-induced softening behaviors of five metals.
\end{abstract}


The current density threshold in EA tension was defined and formulated based on the semi-empirical softening function, which nonlinearly increased with grain size, but decreased with specimen size and electrical resistivity.

Keywords: electrically-assisted, softening behavior, size effect, micro-tension, magnesium alloy, modeling

*Corresponding authors. E-mail address: xjhit@ @it.edu.cn (J. Xu); jcao@ northwestern.edu (J. Cao)

\section{Introduction}

As one of the promising micro-manufacturing techniques for scale production, micro-forming has attracted much attention in the past decade in terms of material, process and modeling, etc [1]. However, the so-called size effect has been found to increasingly lead to deviations in expected material and process performances with miniaturization, including the increase of forming defects as well as the decease of forming limit and precision [2, 3]. Electrically-assisted (EA) forming, i.e., a hybrid process by applying electricity to metals during plastic deformation, has been demonstrated to have technical advantages such as enhanced formability [4, 5], reduced springback [6, 7], increased surface quality and improved geometric accuracy of parts $[6,8]$. Therefore, a possible way to eliminate the size effect is to assist in plastic micro-forming by applying electricity.

A large number of variations in mechanical properties and microstructure of metals caused by electric current such as stress relaxation, creep, residual stress, dislocation generation and mobility, fracture and fatigue, dynamic recovery and dynamic recrystallization (DRX), phase transition, etc., were reviewed by Nguyen-Tran et al. [9]. For example, Li et al. [10] observed various microstructure evolutions including the inhibition of dislocation tangles, the promotion of dislocation glide, the acceleration of DRX and the restraint of cavity/crack growth caused by pulse current, which provided good evidences for the formability improvement of AZ31 magnesium alloy 
during EA gas bulging process. Sánchez et al. [5] found that the drawability of 308L wires increased up to $11.9 \%$ during EA wire drawing process and electropluses could induce DRX, detwinning as well as the attenuation of $\alpha$ martensite. A recent study [4] on the EA multi-pass rolling of magnesium alloy demonstrated that a "twinning-shear banding-DRX" microstructure evolution occurred, causing rollability enhancement, grain refinement and texture alteration. It should be noted that these observations were often attributed to an athermal effect of electricity during EA forming, i.e., the electroplastic effect, termed by some early researchers like Troitskii [11]. In order to account for the electroplastic effect, a well-known mechanism has been usually mentioned in classic works and studies, i.e., the electron-dislocation interaction, leading to a theoretical consideration of the so-called "electron wind force" [12]. However, the athermal effect is complicated and disputable since many recent studies $[13,14]$ claimed that the thermal effect due to Joule heating dominated the EA deformation behavior of various metals, which can be used to model EA flow stress without taking the athermal effect into account. Therefore, more experimental studies associated with EA forming would be needed in future in order to find the main factor and parameter influencing on the electroplastic effect.

It should be noted that most previous researches associated with EA forming mainly focused on the effect of electric pulse parameters on mechanical changes and microstructure evolutions of various metals, while the grain size effect and the specimen size effect on EA deformation behavior were scarcely discussed. Moreover, most EA forming experiments were conducted at the macro-scale level instead of the meso/micro-scale level. More recently, only a few studies on EA micro-forming have been conducted. Wang et al. [15] found that the amount of the Hall-Petch slope decreased in order of the room temperature, the oven-heated and the EA micro-tension tests of AZ31 magnesium alloy. Both the Hall-Petch effects in the EA micro-tension and the air-cooled EA 
micro-tension are insignificant, showing nearly levelled Hall-Petch curves across the grain size regime examined. These EA Hall-Petch behaviors were then modeled and explained based on the assumption of localized Joule heating effect at grain boundaries. Zheng et al. [16] investigated the work hardening, the strain rate sensitivity and the Joule heating of pure titanium foils at various current densities during EA micro-tension, which were included in a derived constitutive model to effectively capture the temperature and flow stress of ultra-thin $\mathrm{Ti}$ foils. Other observations associated with EA micro-forming included: (1) The EA micro-compression flow stress reduction of copper increased with decreasing grain size, increasing prior cold work and current density [17, 18]; (2) The flow stress scatter occurring in micro-compression of copper with miniaturization could be eliminated by increasing current density [17]; (3) The strain distribution through the thickness was more uniform during the EA three-point micro-bending of C260 brass, especially for coarse grain structures, leading to less springback [19]. However, it should be noted that the mechanism of the influence of electric current on size effect is still not clear and the corresponding modeling study is seldom reported and complicated.

The goal of this work is to investigate the effects of grain size and specimen size on the electrically-induced softening behavior in EA micro-forming based on the integral combination of experimental and modeling approaches. To accomplish this, the uniaxial micro-tension tests of AZ31 magnesium alloy specimens subjected to different current densities have been conducted for various grain sizes and specimen sizes. A method is presented to evaluate the influence of electric current on the decrease of tensile strength in EA micro-tension, leading to further characterization of the effects of specimen and grain size on the electrically-induced softening behavior of AZ31. A semi-empirical model of electrically-induced softening behavior with consideration of the size effect in EA micro-tension is proposed to predict the softening behaviors of five engineering metals 
subjected to various current densities during uniaxial tension in literature. Finally, the current density threshold, observed and deemed by many researchers in this field, will be quantified based on the electrically-induced softening function for different grain sizes, specimen sizes and materials, which will help with reducing the trial-and-error process currently desired in EA forming.

\section{Experiment}

\subsection{Specimen preparation}

Commercial drawn AZ31 magnesium alloy rods having a diameter of $15 \mathrm{~mm}$ and the chemical composition of $94.8 \% \mathrm{Mg}, 3.5 \% \mathrm{Al}, 1.2 \% \mathrm{Zn}$ and $0.5 \% \mathrm{Mn}$ were selected as the as-received materials in this study, which were divided into multiple groups for intentionally obtaining various grain sizes by heat treatment and equal-channel angular pressing (ECAP) processes. The microstructures of the as-received, heat-treated and ECAP materials were examined using optical microscope $(\mathrm{OM})$ and scanning electron microscope (SEM) after grinding, polishing, and etching, showing uniform equiaxed grains with the average sizes of $5.4 \pm 0.3 \mu \mathrm{m}, 1.5 \pm 0.13 \mu \mathrm{m}, 7.1 \pm 1.1$ $\mu \mathrm{m}, 17.6 \pm 2.3 \mu \mathrm{m}, 43.3 \pm 4.4 \mu \mathrm{m}$ and $99.8 \pm 24.9 \mu \mathrm{m}$ for the as-received, the ECAP, the $400{ }^{\circ} \mathrm{C}$, $500{ }^{\circ} \mathrm{C}, 550{ }^{\circ} \mathrm{C}$ and $600{ }^{\circ} \mathrm{C}$ annealed samples, respectively. The annealing time was set to be $2 \mathrm{~h}$ for all the samples. The ECAP processing was conducted for 2 and 4 passes at $200{ }^{\circ} \mathrm{C}$ using a die made of $\mathrm{H} 13$ steel with an internal angle of $110^{\circ}$ and an outer arc of curvature of $20^{\circ}$ [20].

Uniaxial micro-tension specimens were cut into dogbone shapes from the axial cross sections of the annealed and ECAP rods along the axial direction using electrical discharge machining [15]. The dogbone samples were prepared in various geometry sizes with the gauge length scaled down by a factor of $\sim 1.5$, but the ratio of gauge length to gauge width maintained at 2.5 , as listed in Table 1. In order to avoid the effect of surface burning and oxidation during sample cutting, the specimens were mechanically polished from an initial cutting thickness of $\sim 0.3 \mathrm{~mm}$ to a final thickness of $\sim 0.2$ 
$\pm 0.05 \mathrm{~mm}$ for micro-tension tests.

Table 1. Specimen sizes of EA uniaxial tension samples.

\begin{tabular}{ccccc}
\hline Sample \# & $\begin{array}{c}\text { Gauge length } \\
(\mathrm{mm})\end{array}$ & $\begin{array}{c}\text { Gauge width } \\
(\mathrm{mm})\end{array}$ & $\begin{array}{c}\text { Overall length } \\
(\mathrm{mm})\end{array}$ & $\begin{array}{c}\text { Width of grip section } \\
(\mathrm{mm})\end{array}$ \\
\hline TS1 & 2.5 & 1 & 14.5 & 5 \\
TS2 & 3.75 & 1.5 & 15.75 & 5 \\
TS3 & 5 & 2 & 17 & 5 \\
TS4 & 7.5 & 3 & 19.5 & 5 \\
\hline \hline
\end{tabular}

Note that the machining tolerance for all the geometry sizes was $\pm 0.1 \mathrm{~mm}$.

\subsection{EA micro-tension system}

The EA micro-tension system was developed on the basis of a commercial desktop tension/compression testing machine (SEMtester 1824LM, MTI Instruments/Fullam Inc.), as illustrated in Fig. 1. It is observed that two precisely aligned lead screws with contrary thread directions connected to the top and bottom crossbeams are driven by a turbo charging system using a DC motor (24 V, Series 2642 CR, Faulhaber). The load cell installed on the upper beam has $100 \mathrm{~N}$ capacity with a resolution of $0.01 \mathrm{~N}$. Two ceramic washers were inserted on the interfaces between EA tension grips and crossbeams to insulate the loading stage from the charged grips during EA tension.

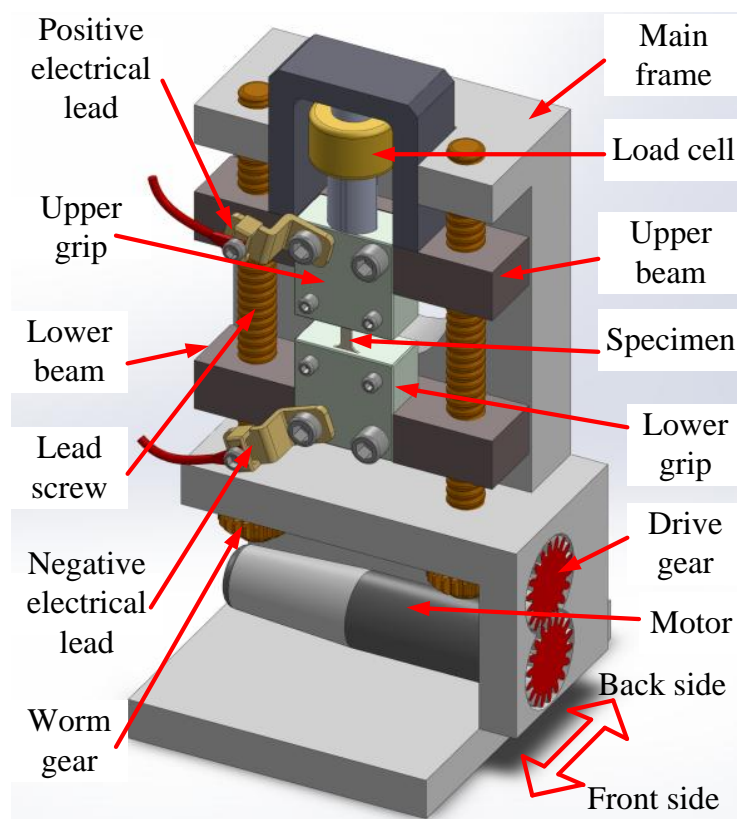

Fig. 1 Schematic illustration of micro tension/compression testing machine. 
Other three subsystems included a rectifier-based DC power supply, a noncontact displacement/strain measurement system and a noncontact temperature measurement system. The power supply (MicroStar CRS-LFP12-300, Dynatronix Inc.) has a maximum output current of 300 $\mathrm{A}$ and a maximum voltage of $12 \mathrm{~V}$, which can output continuous current and low frequency square wave pulse current. As shown in Fig. 1, the positive and negative electrical leads of the power supply were securely fixed to the upper and lower tension grips, respectively, using two Telflon-covered steel screws in order to avoid electrical charging the other units on the test machine. A high resolution CCD camera, located on the front side of the loading stage, was used to capture the images of patterned specimens during EA tension at a frequency of $7.5 \mathrm{~Hz}$. Afterward, the captured images were processed using digital image correlation (DIC) software called Ncorr [21] to obtain the displacement/strain during micro-tension of specimens subjected to electricity. The infrared temperature images of EA tension specimens were captured through a hole on the loading stage's main frame by an infrared camera (spatial resolution: $\sim 170 \mu \mathrm{m} /$ pixel, thermal resolution: $0.1{ }^{\circ} \mathrm{C}$, range: $-20 \sim 900{ }^{\circ} \mathrm{C}$, maximum sampling rate: $120 \mathrm{~Hz}$ ) which was located on the back side of the loading stage. A thin layer of high-temperature pure black paint was sprayed on the back side of EA tension samples to avoid the deviations of infrared temperature measurements causing by the variable emissivity of shiny metallic surfaces. Also, the infrared temperature measurements were initially calibrated by a thermocouple for comparison prior to test.

\subsection{Tensile tests}

In this study, all the tensile tests were monitored by a LabVIEW-based data acquisition and controlling system. Since the power supply was set to output continuous constant-amplitude DC current in this study, the actual current density passing through specimens increased with shrinking cross-section areas during EA tension. Therefore, a nominal current density, i.e., current intensity / 
initial cross-section area, was used in the following context for the comparative study of electrically-induced softening behaviors of AZ31 sheets. Note that the nominal current density varied in the range of $0-214 \mathrm{~A} / \mathrm{mm}^{2}$ depending on the specimen size for each EA tension test in this study. Tensile deformation was controlled to occur after the stabilizing stage (i.e., a few seconds later after turning on electricity and showing little variations of Joule heating temperature with time) rather than before the high-rate heating stage (i.e., within $1 \mathrm{~s}$ after turning on electricity and showing very high Joule heating rate) [14] for all the tests to avoid some additional effects on the mechanical behavior of AZ31 sheets such as thermal expansion. Also, it should be mentioned that all the tests were performed under a strain rate of $0.01 \mathrm{~s}^{-1}$ until fracture and run at least 3 times for repeatability.

\section{Results and discussion}

\subsection{Joule heating temperature}

It was found that the electrically-induced temperature was non-uniformly distributed in the gauge area with the maximum temperature occurring at the center of specimens due to the symmetric configuration of uniaxial tension $[14,15]$. The maximum temperatures during EA tension were averaged for samples with various grain sizes and specimen sizes, which were plotted against the square of current density. As shown in Fig. 2, the average maximum temperature linearly increases with the square of current density in accordance with the Joule heating law. The fit slope of the average maximum temperature against the square of current density decreases with miniaturization, showing the increase rate of TS1 $\sim 0.15$ times that of TS4. This indicates that less electric power is needed to incur the same temperature for larger samples. This may be explained by the result in Fig. 3 that as the increase of the surface area-to-volume ratio of EA tension samples, the average maximum Joule heating temperature nonlinearly deceases since a smaller sample has a 
larger surface area-to-volume ratio, leading to more heat lost.

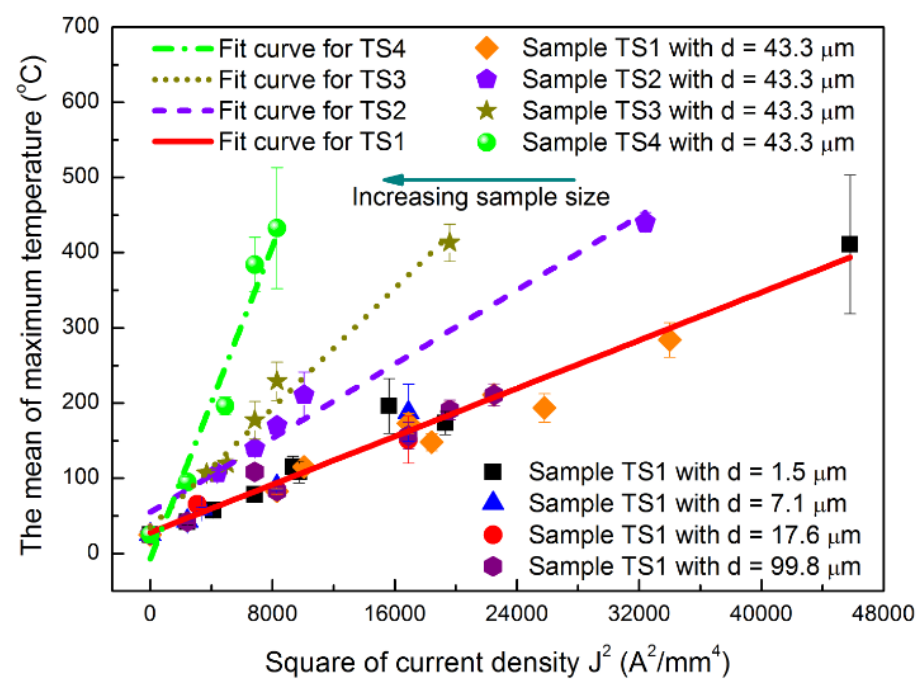

Fig. 2 Relationship between the mean of maximum temperature and the square of current density for various grain size samples with the same specimen size as well as different specimen size samples with the same grain size.

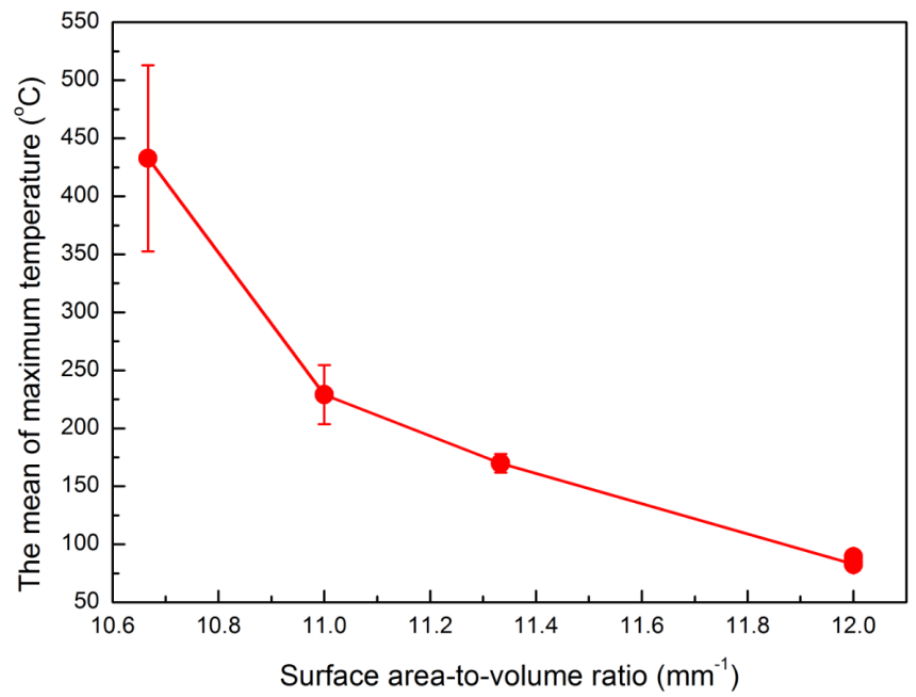

Fig. 3 Relationship between the mean of maximum temperature and the surface area-to-volume ratio of various specimen sizes at $91.1 \mathrm{~A} / \mathrm{mm}^{2}$.

Assuming that all the input electric power is capacitively stored in the sample gauge volume in the form of Joule heating, it gives,

$$
I^{2} R=(J A)^{2} \frac{\rho_{e} L}{A}=c m \frac{\Delta T}{\Delta t}=c \rho_{m} A L \frac{T-T_{R}}{t}
$$


where $I, J, R, \rho_{e}, m, \rho_{m}, c, T, T_{R}, A, L$ and $t$ are current intensity, current density, electrical resistance, electrical resistivity, mass, mass density, specific heat capacity, Joule heating temperature, room temperature, cross-sectional area, gauge length and time, respectively. The maximum Joule heating temperature can be obtained using Eq. (1), that is,

$$
T=\frac{\rho_{e} J^{2}}{c \rho_{m}} t+T_{R}
$$

Equation (2) demonstrates that the maximum Joule heating temperature is proportion to the square of current density just as shown in Fig. 2. However, the conflict between Eq. (2) and the experimental results in Figs. 2 and 3 is that the Joule heating temperature also depends on specimen size. A possible reason for this is that Eq. (1) is based on the assumption of regarding the specimen gauge volume as an object with a uniform temperature distribution, which conflicts with the experimental results $[14,15]$. Besides, the adiabatic condition used in Eq. (1) cannot be practical during EA tension because heat is dissipated through conduction and convection which should be dependent on specimen size in terms of surface area (e.g., Fig. 3) and conduction length. This has been also demonstrated in terms of the thermo-mechanical modeling sensitivity analysis of EA forming by Bunget et al. [22]. Since a parabolic function can be used to simulate the Joule heating temperatures at points $(0, y)$ along specimen gauge length $[13,14]$, it can be written as,

$$
T(y)=T_{R}-a\left(y-\frac{L}{2}\right)\left(y+\frac{L}{2}\right)
$$

based on the assumption that the temperatures at the top end $(0, L / 2)$ and the bottom end $(0,-L / 2)$ in the gauge area are $T_{R} . a$ in Eq. (3) is the magnitude of the quadratic term coefficient which can be collected by the regression analysis of the EA tension data of samples having various specimen sizes at a current density of $91.1 \mathrm{~A} / \mathrm{mm}^{2}$, as shown in Fig. 4. Although the quadratic term coefficient does not change much with specimen size, the maximum temperature at 
center (i.e., $y=0$ ), as shown in Eq. (3), increases with the square of gauge length, causing a specimen size effect on Joule heating temperature in EA tension. Note that the samples with the same specimen size (i.e., TS1) but various grain sizes show almost the same increase rate of Joule heating temperature, which is consistent with the result in literature [15]. It is perhaps because the similar macro-scale temperature, i.e., occurring on the surface of samples, is caused by the similar boundary conditions due to the same specimen size, while the micro-scale temperature such as the Joule heating at grain boundaries inside materials, cannot be captured using the infrared camera in this study.

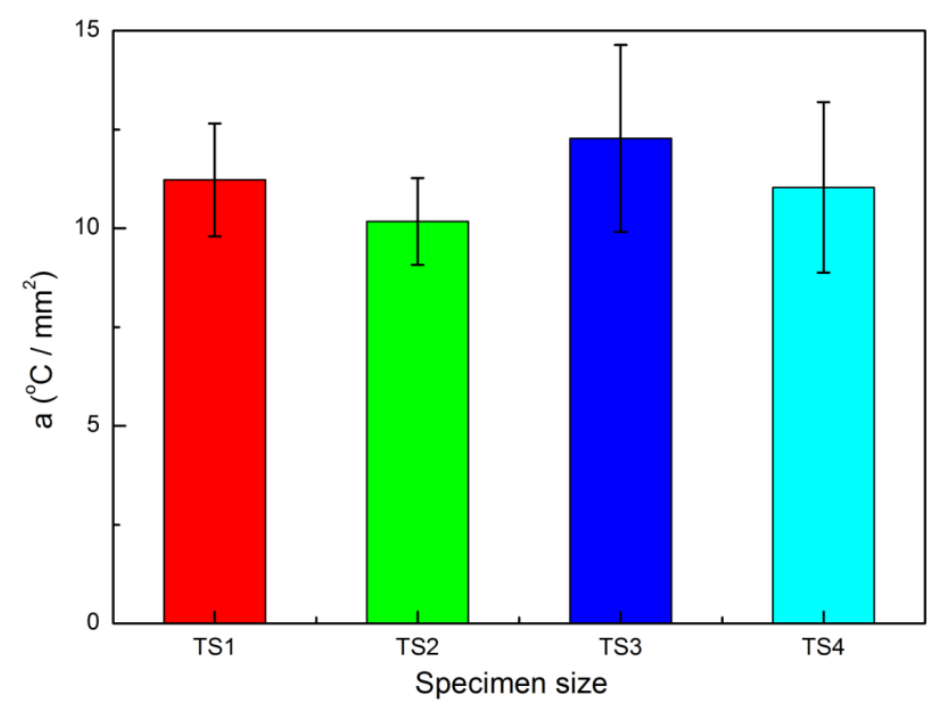

Fig. 4 Variation of the magnitude of quadratic term coefficient with specimen size.

\subsection{Electrically-induced softening parameter}

The engineering stress-strain curves at various current densities for TS1 samples with $\sim 1.5 \mu \mathrm{m}$ grain size are shown in Fig. 5. It can be seen that the EA engineering stress decreases with the increase of current density. Note that the overall engineering strain increases but shows no systematic trend with current density, which might be due to the instability of diffuse necking in EA tension. The tensile strengths of the curves in Fig. 5 are obtained, which are plotted against the current density as shown in Fig. 6. It can be observed that the electrically-induced tensile strength 
decreases slower in the lower current density range but faster in the higher current density range, exhibiting an inverse-S-shaped curve in the overall range of current density in Fig. 6. This trend is similar to the thermal-mechanical behavior occurring during warm/hot tension of metals [13, 23], indicating that the electrically-induced deformation behavior is probably thermally activated.

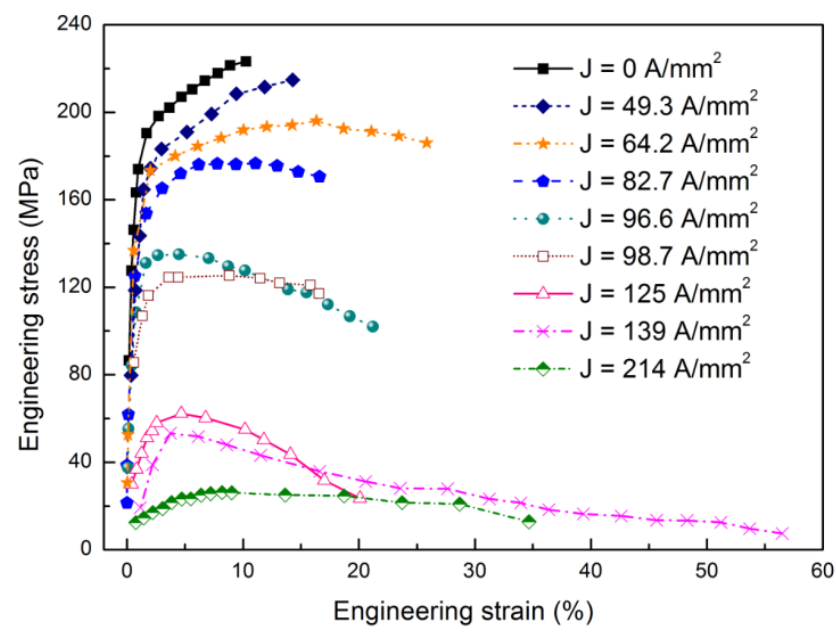

Fig. 5 Engineering tensile stress-strain curves at different current densities for TS1 AZ31 samples.

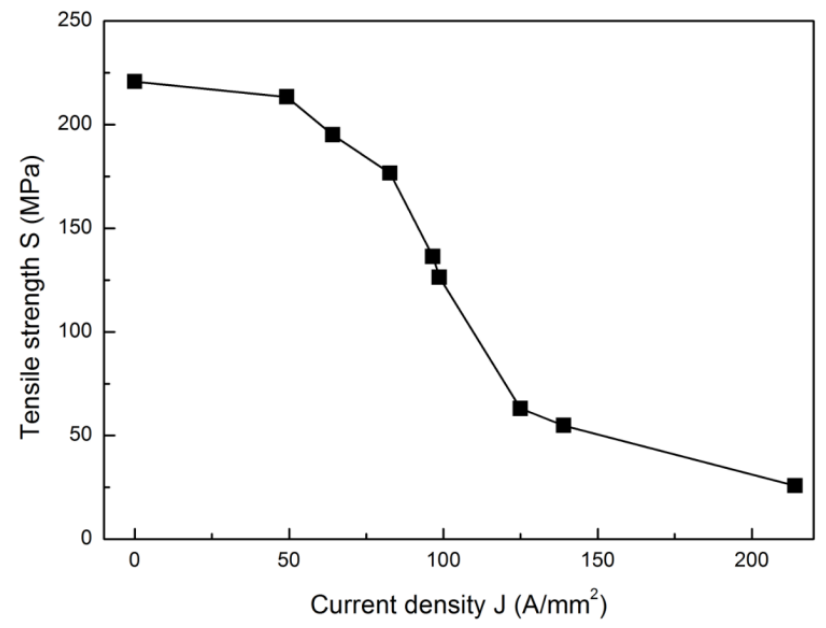

Fig. 6 Relationship between tensile strength and current density for TS1 EA micro-tension samples.

A parameter is usually defined to characterize the softening behavior of metals during warm/hot uniaxial tension, i.e., thermal softening parameter $R[13,23]$, which can be given as,

$$
R=\frac{S_{u}(T)}{S_{u}\left(T_{R}\right)}
$$

where $S_{u}(T)$ and $S_{u}\left(T_{R}\right)$ are tensile strengths at temperature $T$ and room temperature $T_{R}$, 
respectively. It is reported that the thermal softening parameter of low melting temperature metals at elevated temperatures can be expressed by an Arrhenius type exponential function [13, 23], i.e.,

$$
R\left(T_{H}\right)=\frac{\alpha_{T}}{\alpha_{T}+\exp \left(\beta_{T} T_{H}\right)}
$$

where $\alpha_{T}$ and $\beta_{T}$ are empirical constants and $\beta_{T}$ has an average value of $\sim 15$ [23]. Note that the homologous temperature $T_{H}$ can be given as,

$$
T_{H}=\frac{T-T_{R}}{T_{m}-T_{R}}
$$

where $T_{m}$ is the melting temperature of metals.

For EA tension, the temperature is proportional to the square of current density according to the Joule heating law, i.e., Eq. (2). Applying Eq. (2) to Eq. (6), we could have the current-induced homologous temperature $T_{H J}$ as,

$$
T_{H J} \propto \frac{\rho_{e} J^{2}}{c \rho_{m}\left(T_{m}-T_{R}\right)}
$$

We define that the electrically-induced softening parameter $R(J)$ in EA tension satisfies a similar function as Eq. (5), i.e.,

$$
R(J)=\frac{S_{u}(J)}{S_{u}(0)}=\frac{\alpha_{J}}{\alpha_{J}+\exp \left(\beta_{J} J^{2}\right)}+\delta_{J}
$$

where $\alpha_{J}, \beta_{J}$ and $\delta_{J}$ are fitting parameters. Note that Eq. (8) is validated in Fig. 7(a) where the relation between the electrically-induced softening parameter and the current density converted from Fig. 6 can be well fitted using Eq. (8), showing an inverse-S-shaped curve. Through a comparison among Eqs. (5)-(8), $\beta_{J}$ can be given as,

$$
\beta_{J}=\frac{\mu_{J} \beta_{T} \rho_{e}}{c \rho_{m}\left(T_{m}-T_{R}\right)}
$$

where $\mu_{J}$ is a correction function which depends on the effect of experiment/process parameters 
such as specimen size and grain size on Joule heating temperature. In order to determine $\alpha_{J}$ and $\delta_{J}$, two boundary conditions, i.e., $R(0)=1$ and $R(\infty)=0$, are applied to Eq. (8), as given by Eq. (10), i.e.,

$$
\left\{\begin{array}{c}
\alpha_{J} /\left(\alpha_{J}+1\right)=1-\delta_{J} \\
\delta_{J} \rightarrow 0
\end{array}\right.
$$

Note that $\alpha_{J}$ depends on $\delta_{J}$ and $\delta_{J}$ represents the error of fitting of the inverse-S-shaped curve. We take the data in Fig. 7 (b) for example to illustrate the influence of $\delta_{J}$ on the electrically-induced softening parameter, which shows various fit curves using Eq. (8) when holding $\beta_{J}$ constant at $2.35 \times 10^{-4}$ but varying $\delta_{J}$ and $\alpha_{J}$. It can be seen that the "tail" and "head" parts of the curves move upward with increasing $\delta_{J}$. However, the combinations of $\delta_{J}$ and $\alpha_{J}$ do not change the "body" part (i.e., the interesting electrically-induced softening area) of the curves much when varying $\delta_{J}$ in the range of $1 \times 10^{-3} \sim 0.079$. Therefore, in the following study, we assume $0 \leq \delta_{J} \leq 0.079$, which is used as a uniform boundary condition of Eq. (8) for simplification in order to fit the relationships between electrically-induced softening parameter and current density for various grain sizes as well as various specimen sizes.
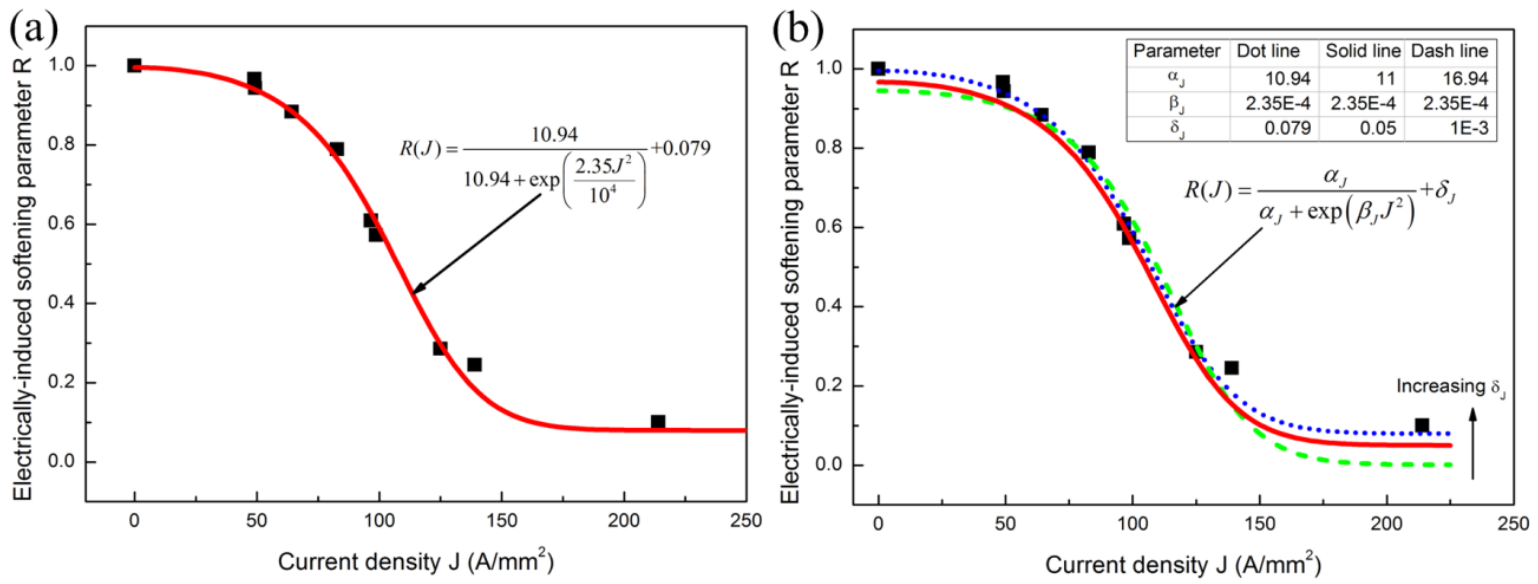

Fig. 7 (a) Relationship between electrically-induced softening parameter and current density of AZ31 samples in EA micro-tension; (b) Inverse-S-shaped curves fitted using various combinations of parameter values. 
The variations of electrically-induced softening parameter with current density for different grain sizes and specimen sizes of AZ31 samples in EA micro-tension are shown in Fig. 8 (a) and Fig. 8 (b), respectively. As it can be seen, the electrically-induced softening parameter is not only dependent on current density, but also affected by grain size and specimen size. The inverse-S-shaped fit curve shifts to the higher and the lower current density regions with the increases of grain size and specimen size, respectively, indicating that a lower electrical energy would be sufficient to achieve a higher softening effect for the smaller grain size and the larger specimen size samples during EA tension. Specifically for EA micro-forming, fine grained and high electrical resistivity material would be preferred since generally more electrical energy is required to soften miniaturized specimens.
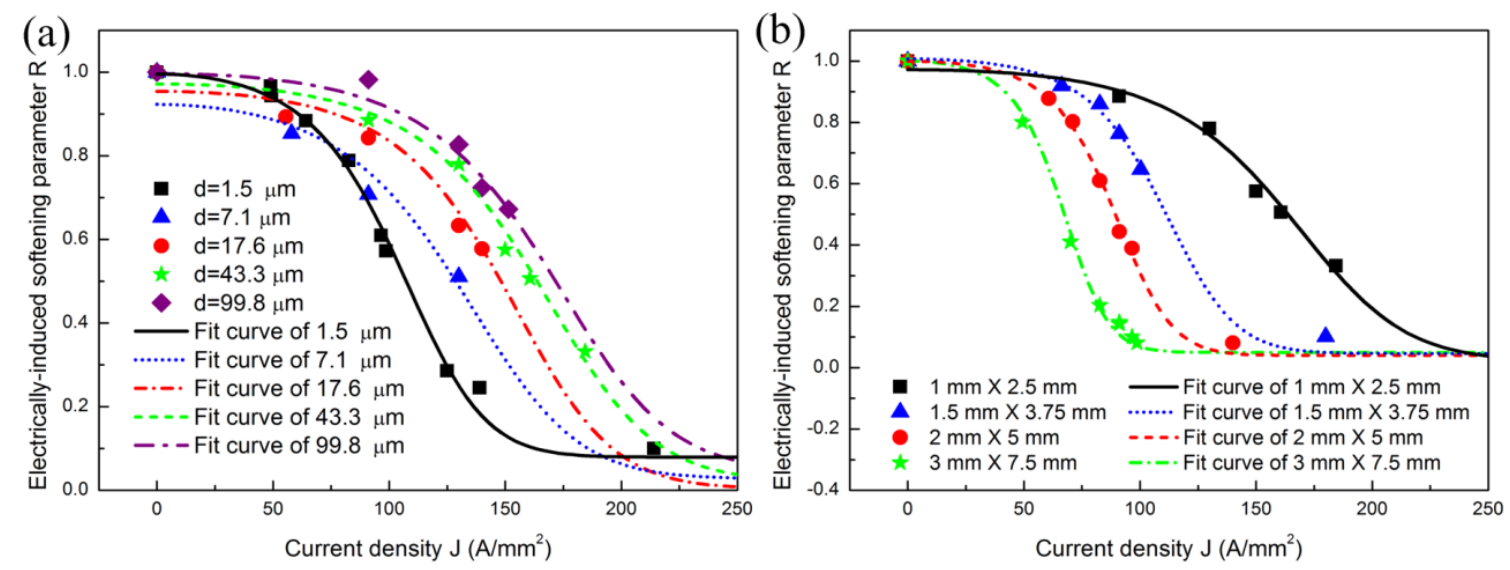

Fig. 8 Variations of electrically-induced softening parameter with current density for different (a) grain sizes and (b) specimen sizes of AZ31 samples in EA micro-tension.

In order to quantify the interactive effect of the specimen and grain sizes on the electrically-induced softening parameter, a parameter associated with the ratio of specimen size to grain size, i.e., grain number in specimen gauge volume $N$, is introduced as,

$$
N=\frac{V_{g}}{V_{d}}
$$


where $V_{d}$ and $V_{g}$ are grain volume and specimen gauge volume, respectively. As shown in Fig. 9, the electrically-induced softening parameter at a current density of $91.1 \mathrm{~A} / \mathrm{mm}^{2}$ is found to linearly decrease with the logarithm of the grain number in specimen gauge volume. The negative slope when increasing specimen size is $~ 10$ times that obtained by decreasing grain size, indicating that the electrically-induced parameter is more sensitive to specimen size. It is of interest to note that the size effects on electrically-induced softening behavior may be associated with some microstructural variables in materials since dislocation density and the quantity of micro-voids have been demonstrated to correlate with the grain number $N$ [24].

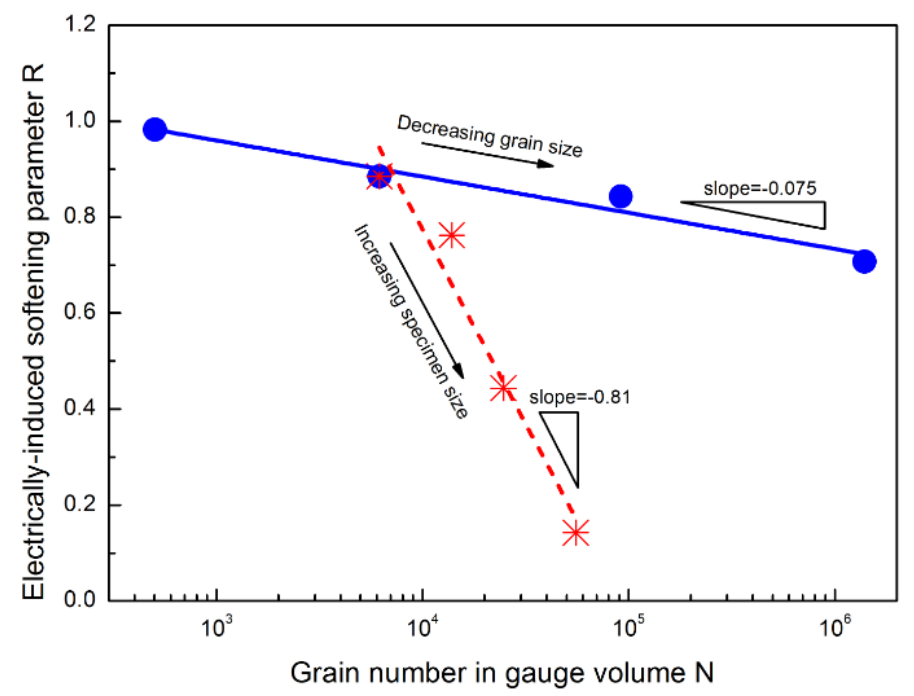

Fig. 9 Variation of electrically-induced softening parameter at $91.1 \mathrm{~A} / \mathrm{mm}^{2}$ with grain number in specimen gauge volume for different grain sizes and specimen sizes.

\subsection{Semi-empirical modeling of electrically-induced softening behavior}

It should be noted that the offset behavior of the inverse-S-shaped curve in Fig. 8 is dependent on $\beta_{J}$ according to Eq. (9) and Eq. (10) which can be named as offset coefficient. Specifically, the inverse-S-shaped curve moves to the left side with increasing the offset coefficient, causing a more efficient Joule heating softening effect, and vice versa. The variations of the offset coefficient with grain volume $V_{d}$ and specimen gauge volume $V_{g}$ in Fig. 10 (a) and Fig. 10 (b), respectively, show 
that $\beta_{J}$ nonlinearly decreases with the grain size but increases with the specimen size. These size effects on the offset coefficient can be well fitted by power functions as,

$$
\beta_{J}=\left\{\begin{array}{cc}
\frac{5.74 V_{d}^{-0.0698}}{10^{5}} & \left(V_{g}=0.5 \mathrm{~mm}^{3}\right) \\
\frac{2.416 V_{g}^{0.708}}{10^{4}} & \left(V_{d}=d^{3}=8.12 \times 10^{-5} \mathrm{~mm}^{3}\right)
\end{array}\right.
$$
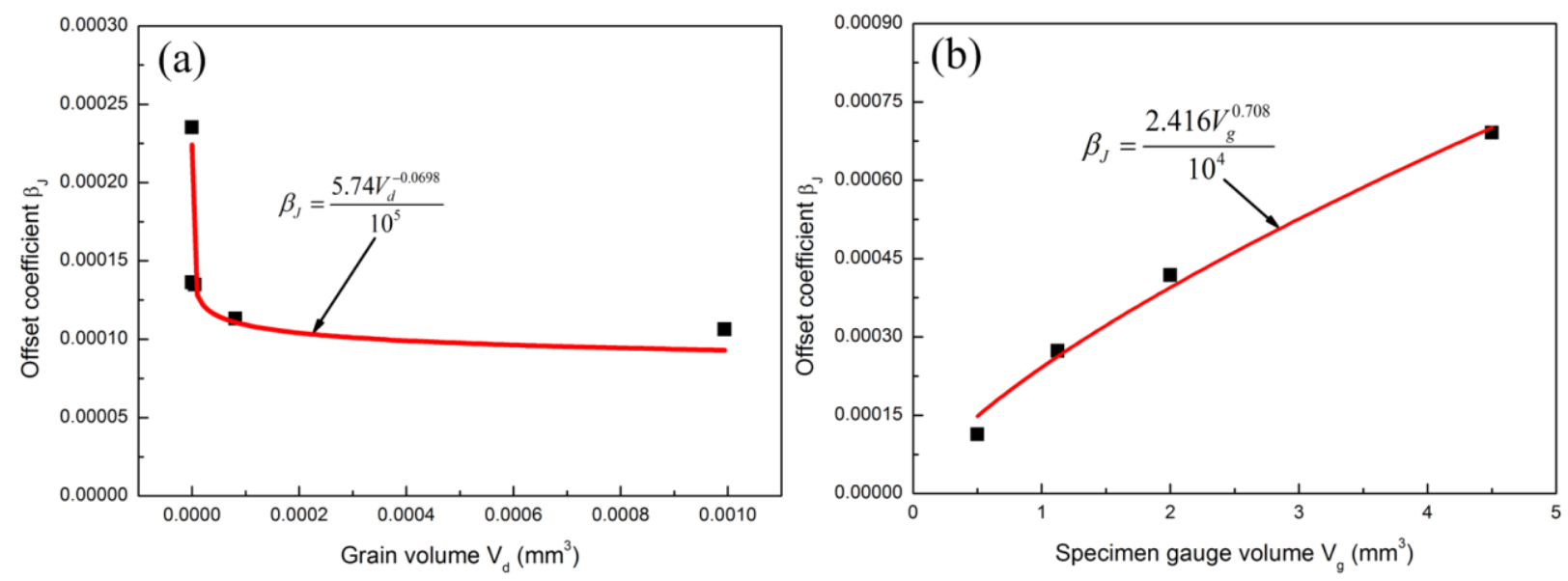

Fig. 10 Variations of offset coefficient with (a) grain volume and (b) specimen gauge volume.

Since the data in Fig. 8 (a) and Fig. 8 (b) were obtained during the EA tension of TS1 samples and 43.3 $\mu \mathrm{m}$ grain size samples, respectively, the two power functions in Eq. (12) would be valid only when $V_{g}=0.5 \mathrm{~mm}^{3}$ and $V_{d}=8.12 \times 10^{-5} \mathrm{~mm}^{3}$, respectively. Another interesting point to be noted in Fig. 8 is that $\alpha_{J}$ does not show significant variations for different grain sizes and specimen sizes with an average value of $14.9 \pm 3.8$, which implies that $\alpha_{J}$ should be a material-dependent parameter. An intuitive estimate is that $\alpha_{J}$ should be associated with thermal conductivity $k$ since metals with a higher thermal conductivity would generally need more electrical current to heat them up due to more conductive heat loss. Besides, a larger cross-sectional area and a smaller conduction length would help to increase heat transfer rate, causing a relatively lower Joule heating efficiency. Note that the ratio of cross-sectional area $A$ to conduction length $L$ for all the specimens in this study is the same, i.e., $0.08 \mathrm{~mm}$, which may be the reason for the relatively 
consistent $\alpha_{J}$ obtained above for all the specimens. Therefore, we assume that $\alpha_{J}$ can be written as,

$$
\alpha_{J}=\xi \frac{k A}{L}
$$

where $\xi$ is a constant which can be calculated as 2.1 using the average value of $\alpha_{J}$, the thermal conductivity of AZ31 (as listed in Table 2) and the area-to-length ratio of the samples in this study.

We assume that $\mu_{J}$ in Eq. (9) is only the function of grain volume and specimen gauge volume and the effects of other experiment/process parameters are neglected. Since grain size and specimen size are two independent variables as well as $\mu_{J}$ has the similar function type as that of $\beta_{J}$, we can express $\mu_{J}$ as,

$$
\mu_{J}\left(V_{d}, V_{g}\right)=\lambda_{\mu} V_{g}^{0.708} V_{d}^{-0.0698}
$$

where $\lambda_{\mu}$ is a correction factor. It should be noted that $\mu_{J}$ may reflect the Joule heating effect at grain boundaries [15] on material softening to some extent since the combining form of $V_{g}$ and $V_{d}$ in Eq. (14) is somehow associated with the grain number $N$ in specimen gauge volume, i.e., Eq. (11). Considering the boundary conditions, i.e., Eq. (12), we can calculate $\lambda_{\mu}$ in Eq. (14) based on Eq. (9), that is,

$$
\mu_{J}\left(V_{d}, V_{g}\right)=\left\{\begin{array}{cc}
\lambda_{\mu} 0.5^{0.708} V_{d}^{-0.0698}=\zeta_{A Z 31} \frac{5.74 V_{d}^{-0.0698}}{10^{5}} & \left(V_{g}=0.5 \mathrm{~mm}^{3}\right) \\
\lambda_{\mu} V_{g}^{0.708}\left(8.12 \times 10^{-5}\right)^{-0.0698}=\zeta_{A Z 31} \frac{2.416 V_{g}^{0.708}}{10^{4}} & \left(V_{d}=8.12 \times 10^{-5} \mathrm{~mm}^{3}\right)
\end{array}\right.
$$

where $\zeta_{A Z 31}=\left[c \rho_{m}\left(T_{m}-T_{R}\right) / \beta_{T} \rho_{e}\right]_{A Z 31}$, represents a constant computed using the physical parameters of magnesium alloy AZ31 as listed in Table 2. The correction factors obtained from the two boundary conditions in Eq. (15) have the same order of magnitude but different values which may be due to the errors of experiment data and curve fitting, i.e., 0.0728 and 0.0971 . An average on these data is used as the value of $\lambda_{\mu}$ in this study, i.e., 0.085. As a result, we can use Eq. (14) to 
modify the current-induced homologous temperature, i.e., Eq. (7), which can be further used along with Eq. (13) to calculate the electrically-induced softening parameter in EA tension of samples with various grain sizes and specimen sizes, i.e.,

$$
R(J)=\frac{\xi k A / L}{(\xi k A / L)+\exp \left\{\mu_{J}\left(V_{d}, V_{g}\right) \rho_{e} J^{2} /\left[c \rho_{m}\left(T_{m}-T_{R}\right)\right]\right\}}+\delta_{J}
$$

where $\delta_{J}$ can be calculated using Eq. (10) and Eq. (13).

Table 2. Material properties of common engineering metals.

\begin{tabular}{llllll}
\hline \hline Material & $\begin{array}{l}\text { Mass } \\
\text { density } \rho_{m} \\
\left(\mathrm{~kg} \mathrm{~m}^{-3}\right)\end{array}$ & $\begin{array}{l}\text { Specific heat } \\
\text { capacity } \\
\left(\mathrm{J} \mathrm{kg}^{-1} \mathrm{C}^{-1}\right)\end{array}$ & $\begin{array}{l}\text { Electrical } \\
\text { resistivity } \\
\rho_{e}(\mathrm{n} \Omega \mathrm{m})\end{array}$ & $\begin{array}{l}\text { Melting temperature } \\
T_{m}\left({ }^{\circ} \mathrm{C}\right)\end{array}$ & $\begin{array}{l}\text { Thermal } \\
\text { conductivity } \\
k\left(\mathrm{~W} \mathrm{~m}^{-1} \mathrm{C}^{-1}\right)\end{array}$ \\
\hline Ti6A14V & 4.43 & 526 & 1780 & 1660 & 7.95 \\
AISI304 & 8.03 & 500 & 720 & 1454 & 13.4 \\
A12024 & 2.78 & 920 & 45 & 638 & 136 \\
Brass & 8.53 & 375 & 62 & 916 & 108 \\
Copper & 8.92 & 385 & 17 & 1084 & 393 \\
AZ31 & 1.77 & 1000 & 92 & 630 & 88.7 \\
\hline \hline
\end{tabular}

*Note that all the values of the parameters are obtained from literature $[23,25,26]$.

As shown in Fig. 11, the electrically-induced softening parameters of various metals at different current densities obtained from the EA tension data of Ross et al. [27] are used to verify the effectiveness of Eq. (16). It should be noted that round bars with $7.9 \mathrm{~mm}$ diameter and $78.7 \mathrm{~mm}$ length were used as EA tension specimens. However, the grain sizes of the specimens were not examined by Ross et al. [27]. A possible method is to utilize the well-known Hall-Petch relationship [28] for the reverse calculation of the grain size $d$ of various metals, that is,

$$
d=\left(\frac{k_{h p}}{\sigma_{Y}-\sigma_{0}}\right)^{2}
$$

where $k_{h p}, \sigma_{Y}$ and $\sigma_{0}$ are Hall-Petch slope, lattice friction stress and yield stress, respectively. As listed in Table 3, the Hall-Petch slopes and the lattice friction stresses can be obtained from literature [29, 30], while the yield stresses can be directly captured from the stress-strain curves of different alloys in [27]. Therefore, the grain sizes for various metals are predicted using Eq. (17) 
with their values listed in the last column of Table 3 .

Table 3. Hall-Petch coefficients and predicted grain sizes of different materials.

\begin{tabular}{lllll}
\hline \hline \multirow{2}{*}{ Material } & $\begin{array}{l}\text { Hall-Petch slope } \\
k_{h p}\left(\mathrm{MPa} \mu \mathrm{m}^{1 / 2}\right)\end{array}$ & $\begin{array}{l}\text { Lattice friction stress } \\
\sigma_{0}(\mathrm{MPa})\end{array}$ & $\begin{array}{l}\text { Yield stress } \\
\sigma_{Y}(\mathrm{MPa})\end{array}$ & $\begin{array}{l}\text { Predicted grain size } \\
d(\mu \mathrm{m})\end{array}$ \\
\hline Ti6Al4V & 190 & 340.8 & $610^{\mathrm{b}}$ & 0.5 \\
AISI304 & 270 & 200 & $520^{\mathrm{b}}$ & 0.71 \\
Al2024 & $156.5^{\mathrm{a}}$ & $94.6^{\mathrm{a}}$ & $280^{\mathrm{b}}$ & 0.71 \\
Brass & 317.5 & $133^{\mathrm{b}}$ & $320^{\mathrm{b}}$ & 2.88 \\
Copper & 160.8 & 90 & $350^{\mathrm{b}}$ & 0.38 \\
\hline \hline
\end{tabular}

Note: ${ }^{a}$ the Hall-Petch coefficients of A12024 obtained from [30], ${ }^{b}$ the yield stresses obtained from [27], and the other values of the coefficients are obtained from [29].

Table 4. Predicted parameters of electrically-induced softening function for various materials.

\begin{tabular}{llll}
\hline Material & $\alpha_{J}$ & $\beta_{J}$ & $\delta_{J}$ \\
\hline Ti6A14V & 10.4 & 0.0675 & 0.09 \\
AISI304 & 17.6 & 0.0168 & 0.05 \\
Al2024 & 178.2 & 0.0039 & 0.006 \\
Brass & 141.6 & 0.0022 & 0.007 \\
Copper & 515.1 & 0.000715 & 0.002 \\
\hline \hline
\end{tabular}

In order to predict the electrically-induced softening behaviors for the materials in [27], $\alpha_{J}$, $\beta_{J}$ and $\delta_{J}$ are calculated using the above equations and the coefficients in Table 2 and Table 3, as summarized in Table 4. It can be seen in Fig. 11 that the overall predictions of the electrically-induced softening behaviors of the five metals using Eq. (16) can effectively match with the experiment data of Ross et al. [27], although the lower values of $R$ are limited due to the lack of experiment data. More EA tension experiments for different materials will be conducted in the future to continuously investigate and improve the model. Note that the predictions for some metals such as Al2024 are not very accurate, which is probably due to the following reasons:

(1) Equation (14) is calibrated under the experiment conditions in this study, while the experiment conditions in [27] are quite different, e.g., the round bar specimen with a conventional specimen size in [27] versus the thin sheet specimen with a miniaturized specimen size in this study; 
(2) The grain sizes listed in Table 3 may be underestimated since $\sigma_{0}$ is typically dependent on Peierls-Nabarro stress, solid strengthening and work-hardening which are affected by processing history and may be underestimated for the commercial materials in [27];

(3) The initial material conditions are also different, e.g., three Al2024 tempers were tested including T4, T351 and cold work hardening in [27] which may influence on the EA softening behavior due to dislocation density and impurity concentration, etc.

Additionally, we plot the predicted curves of Magargee et al. [23] for a comparison in Fig. 11. As it can be seen, the predictions by Magargee et al. [23] deviate much more from the experiment results for all the materials compared to ours, exhibiting much slower electrically-induced softening. The reasons for this may include:

(1) Effects of specimen and grain size on the current-induced homologous temperature $T_{H J}$ were not mentioned in [23];

(2) The parameters of the softening function in [23] were obtained based on the regression analysis of thermal softening parameters in conventional warm/hot tension tests without the consideration of thermal conduction occurring in EA tension.

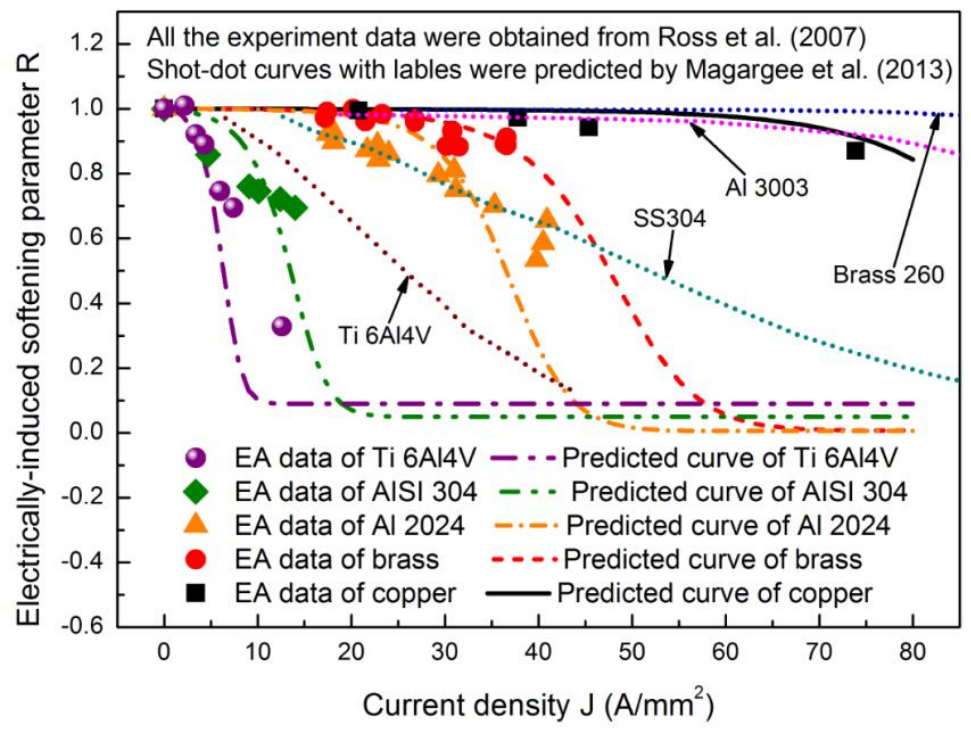

Fig. 11 Comparison between experimental (obtained from Ross et al. [27]) and predicted 
electrically-induced softening parameters (obtained by us and Magargee et al. [23]) at different current densities for various metals.

\subsection{Current density threshold}

Many researchers $[17,31]$ found that electrical current did not significantly alter material deformation behavior below a certain current density, while minor density changes significantly altered material properties above it, which is usually deemed as "current density threshold". These observations are clearly evident when observing the inverse-S-shaped electrically-induced softening behavior. As shown in Fig. 8, a very small softening rate is observed in the lower current density region, while a faster softening occurs in a nearly linear relationship with current density above the current density threshold. In order to quantify the current density threshold, it should be reasonably defined based on the inverse-S-shaped exponential function.

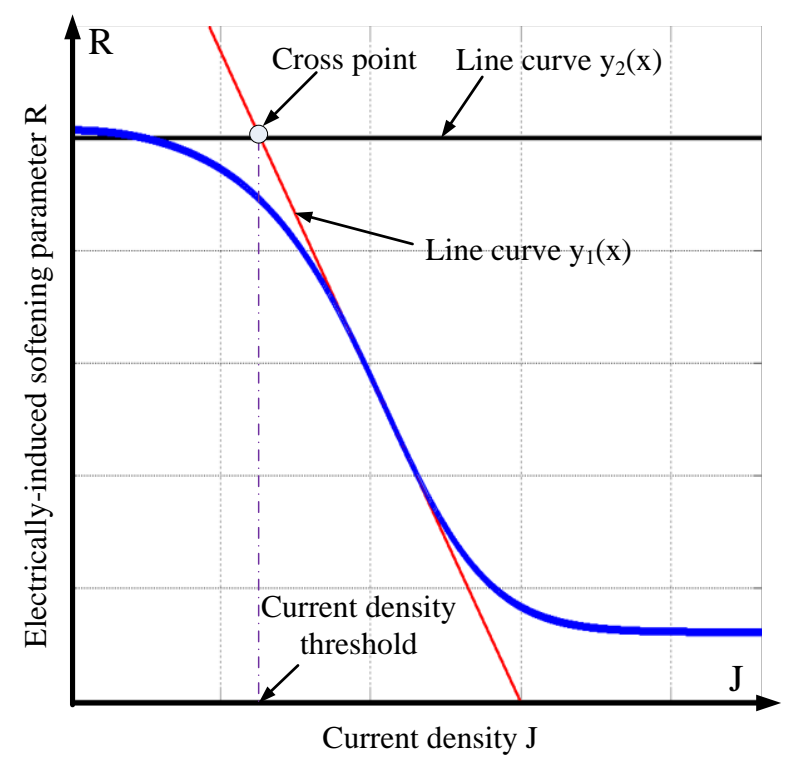

Fig. 12 Schematic illustration of the definition of current density threshold in EA tension.

As shown in Fig. 12, the tangent lines $y_{1}(x)$ and $y_{2}(x)$ are drawn through the points of the minimum derivative and the starting point of the inverse-S-shaped curve, respectively, which can be expressed as, 


$$
\left\{\begin{array}{c}
y_{1}(x)=R_{\min }^{\prime}\left(x-R^{\prime(-1)}\left(R_{\min }^{\prime}\right)\right)+R\left(R^{\prime(-1)}\left(R_{\min }^{\prime}\right)\right) \\
y_{2}(x)=R_{0}^{\prime} x+1
\end{array}\right.
$$

where $R_{\min }^{\prime}$ and $R_{0}^{\prime}$ are the minimum first-derivative and the first-derivative at the starting point of the inverse-S-shaped function, respectively, $R^{(-1)}$ is the inverse of the first derivative of the inverse-S-shaped function. The current density threshold can be quantified by solving the current density coordinate of the crossing point of the curves $y_{1}(x)$ and $y_{2}(x)$, that is,

$$
J_{c}=\frac{R\left(R^{\prime(-1)}\left(R_{\min }^{\prime}\right)\right)-R_{\min }^{\prime} R^{\prime(-1)}\left(R_{\min }^{\prime}\right)-1}{R_{0}^{\prime}-R_{\min }^{\prime}}
$$

According to Eq. (19), the current density thresholds in Fig. 8 (a) and Fig. 8 (b) are obtained, which are plotted against grain volume and specimen gauge volume, respectively, as shown in Fig. 13 (a) and Fig. 13 (b). It can be seen that the current density threshold nonlinearly increases with grain size but decreases with specimen size. These size effects on the current density threshold are fitted using power functions as,

$$
J_{c}=\left\{\begin{array}{cc}
180.5 V_{d}^{0.0548} & V_{g}=0.5 \mathrm{~mm}^{3} \\
83 V_{g}^{-0.4} & V_{d}=8.12 \times 10^{-5} \mathrm{~mm}^{3}
\end{array}\right.
$$
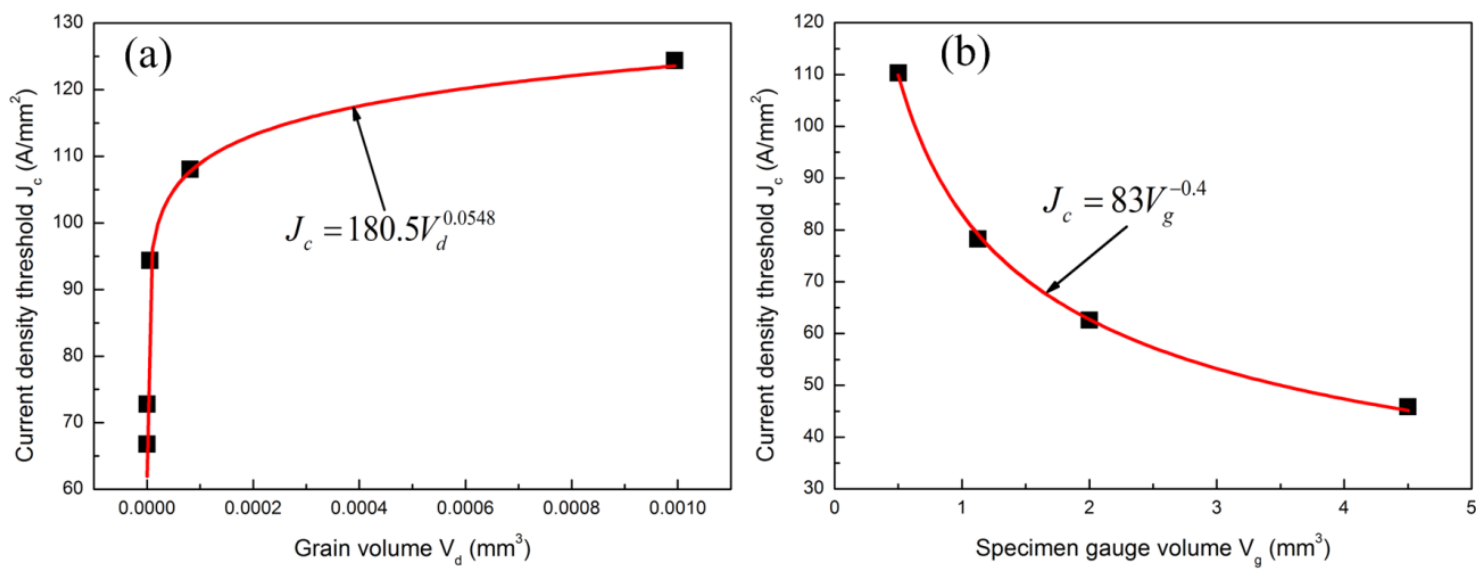

Fig. 13 Variations of current density threshold with (a) grain volume and (b) specimen gauge volume in EA micro-tension of AZ31 magnesium alloy.

Note that Eq. (20) has practical significance to EA forming since engineers can directly calculate current density threshold when providing grain size and specimen size of materials without a costly 
trial-and-error. However, Eq. (20) only determines the current density threshold under two boundary conditions of $V_{g}=0.5 \mathrm{~mm}^{3}$ and $V_{d}=8.12 \times 10^{-5} \mathrm{~mm}^{3}$. More experiment data would be needed in future to further calibrate and determine the general function of the current density threshold with grain size and specimen size.

The current density thresholds of various metals in Fig. 11 can be also calculated using Eq. (19) based on the experiment data of Ross et al. [27], as shown in Fig. 14 (a). It is observed that a minor electrical resistivity increase could cause a significant decrease of current density threshold over the smaller electrical resistivity range, while the current density threshold varies little at the higher electrical resistivity. For example, the current density threshold decreases from $\sim 75 \mathrm{~A} / \mathrm{mm}^{2}$ for copper to $\sim 30 \mathrm{~A} / \mathrm{mm}^{2}$ for $\mathrm{A} 12024$ when changing the electrical resistivity of $17 \mathrm{n} \Omega \mathrm{m}$ for copper to $45 \mathrm{n} \Omega \mathrm{m}$ for A12024. The current density threshold of Ti6Al4V is comparable to that of AISI304, i.e., $\sim 10 \mathrm{~A} / \mathrm{mm}^{2}$, which is consistent with the result of Salandro et al. [32], even though their electrical resistivity difference is up to $1060 \mathrm{n} \Omega \mathrm{m}$. It should be noted that the predicted current density thresholds for these metals based on Eq. (16) and Eq. (19) are also depicted in Fig. 14 (a) using solid marks. As it can be seen, the predicted data match well with the experimental data with the predicted errors, i.e., $J_{c}^{\text {cal }}-J_{c}^{\text {exp }}$, as shown in Fig. 14 (b), falling in the range of $\pm 4 \mathrm{~A} / \mathrm{mm}^{2}$.
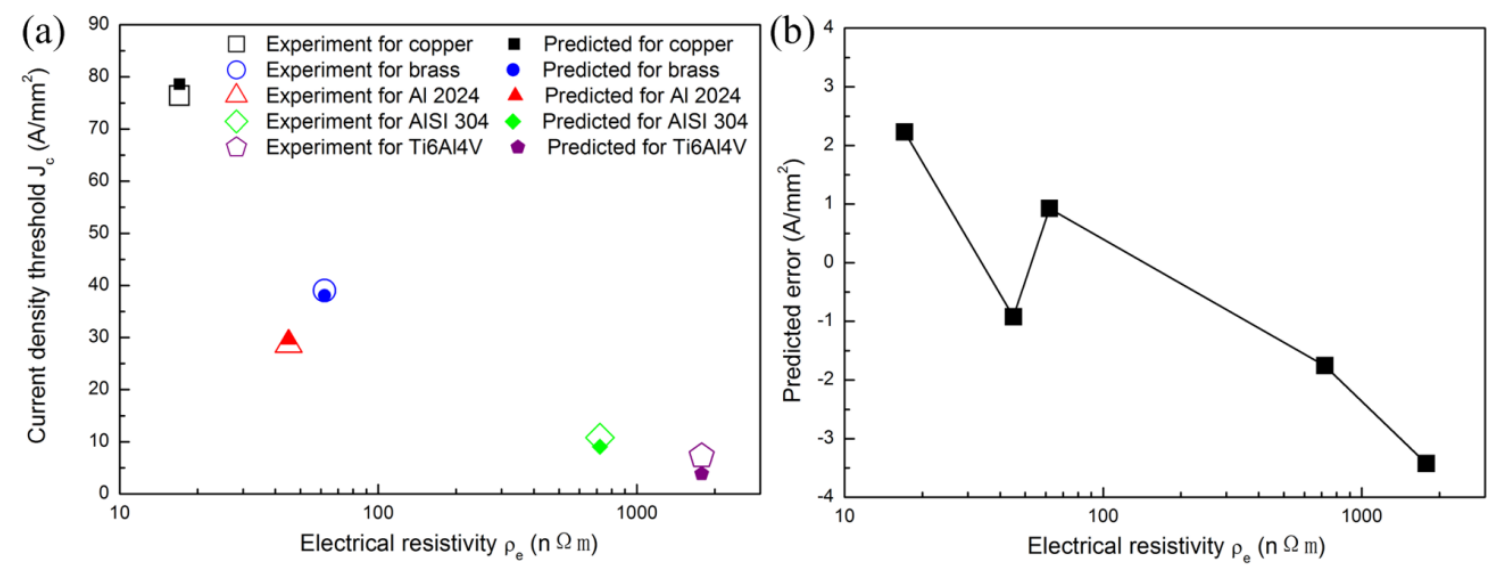

Fig. 14 Relationship between current density threshold and electrical resistivity: (a) a comparison 
between experimental and predicted values of current density threshold with their corresponding predicted errors in (b).

\section{Conclusions}

In this study, the effects of grain size and specimen size on electrically-induced softening behavior were investigated by the uniaxial micro-tension tests of magnesium alloy AZ31 specimens subjected to various current densities, which was used to propose a semi-empirical model of electrically-induced softening behavior. The following conclusions are drawn from this research:

(1) The increasing rate of average maximum temperature against the square of current density decreased with miniaturization but unchanged with grain size.

(2) An inverse-S-shaped decay curve was observed for the relationship between electrically-induced softening parameter and current density, which shifted to the higher and the lower current density regions with the increases of grain size and specimen size, respectively.

(3) A semi-empirical modeling for electrically-induced softening behavior was proposed with consideration of the size effect on inverse-S-shaped function parameters, which was verified by the EA tension data of five engineering metals subjected to various current densities in literature.

(4) The current density threshold can be reasonably defined and quantified based on the semi-empirical model of electrically-induced softening behavior, which was calculated to nonlinearly increase with grain size, but decrease with specimen size and electrical resistivity of materials.

\section{Acknowledgments}

This work was supported by the National Natural Science Foundation of China under Grant No. 51475124 and No. 51635005, and the National Science Foundation of the United States under Grants No. CMMI-1100787 and DMR-1121262. 


\section{Reference}

[1] M.W. Fu, J.L. Wang, A.M. Korsunsky, A review of geometrical and microstructural size effects in micro-scale deformation processing of metallic alloy components, International Journal of Machine Tools \& Manufacture 109 (2016) 94-125.

[2] F. Vollertsen, D. Biermann, H.N. Hansen, I.S. Jawahir, K. Kuzman, Size effects in manufacturing of metallic components, CIRP Annals - Manufacturing Technology 58(2) (2009) 566-587.

[3] N. Krishnan, J. Cao, K. Dohda, Study of the size effect on friction conditions in microextrusion-part I: microextrusion experiments and analysis, Journal of Manufacturing Science and Engineering 129(4) (2007) 669-676.

[4] J. Kuang, X. Li, R. Zhang, Y. Ye, A.A. Luo, G. Tang, Enhanced rollability of Mg 3Al 1Zn alloy by pulsed electric current: a comparative study, Materials \& Design 100 (2016) 204-216.

[5] A.J.S. Egea, H.A.G. Rojas, D.J. Celentano, J.J. Peiró, Mechanical and metallurgical changes on 308L wires drawn by electropulses, Materials \& Design 90 (2016) 1159-1169.

[6] D. Xu, B. Lu, T. Cao, H. Zhang, J. Chen, H. Long, J. Cao, Enhancement of process capabilities in electrically-assisted double sided incremental forming, Materials \& Design 92 (2016) 268-280.

[7] A.J.S. Egea, H.A.G. Rojas, D.J. Celentano, J.A. Travieso-Rodríguez, J.L. i Fuentes, Electroplasticity-assisted bottom bending process, Journal of Materials Processing Technology 214(11) (2014) 2261-2267.

[8] B. Valoppi, A.J. Sánchez Egea, Z. Zhang, H.A. González Rojas, A. Ghiotti, S. Bruschi, J. Cao, A hybrid mixed double-sided incremental forming method for forming Ti6Al4V alloy, CIRP Annals - Manufacturing Technology 65(1) (2016) 309-312.

[9] H.-D. Nguyen-Tran, H.-S. Oh, S.-T. Hong, H.N. Han, J. Cao, S.-H. Ahn, D.-M. Chun, A review of electrically-assisted manufacturing, International Journal of Precision Engineering and Manufacturing-Green Technology 2(4) (2015) 365-376.

[10] C. Li, K. Zhang, S. Jiang, Z. Zhao, Pulse current auxiliary bulging and deformation mechanism of AZ31 magnesium alloy, Materials \& Design 34 (2012) 170-178.

[11] O.A. Troitskii, Electromechanical Effect in Metals, ZhETF Pis. Red 10(1) (1969) 18-22.

[12] A. Sprecher, S. Mannan, H. Conrad, Overview no. 49: On the mechanisms for the electroplastic effect in metals, Acta Metallurgica 34(7) (1986) 1145-1162.

[13] J. Magargee, F. Morestin, J. Cao, Characterization of flow stress for commercially pure titanium subjected to electrically assisted deformation, Journal of Engineering Materials and Technology 135(4) (2013) 041003.

[14] X. Wang, J. Xu, D. Shan, B. Guo, J. Cao, Modeling of thermal and mechanical behavior of a magnesium alloy AZ31 during electrically-assisted micro-tension, International Journal of Plasticity 85 (2016) 230-257.

[15] X. Wang, J. Xu, Z. Jiang, W.-L. Zhu, D. Shan, B. Guo, J. Cao, Size effects on flow stress behavior during electrically-assisted micro-tension in a magnesium alloy AZ31, Materials Science and Engineering: A 659 (2016) 215-224.

[16] Q. Zheng, T. Shimizu, T. Shiratori, M. Yang, Tensile properties and constitutive model of ultrathin pure titanium foils at elevated temperatures in microforming assisted by resistance heating method, Materials \& Design 63 (2014) 389-397.

[17] M.S. Siopis, B.L. Kinsey, Experimental investigation of grain and specimen size effects during electrical-assisted forming, Journal of Manufacturing Science and Engineering 132(2) (2010) 021004.

[18] M.S. Siopis, B.L. Kinsey, N. Kota, O.B. Ozdoganlar, Effect of severe prior deformation on electrical-assisted compression of copper specimens, Journal of Manufacturing Science and Engineering 133(6) (2011) 064502.

[19] A. Jordan, B.L. Kinsey, Investigation of thermal and mechanical effects during electrically-assisted microbending, Journal of Materials Processing Technology 221 (2015) 1-12.

[20] J. Xu, M. Shirooyeh, J. Wongsa-Ngam, D. Shan, B. Guo, T.G. Langdon, Hardness homogeneity and micro-tensile 
behavior in a magnesium AZ31 alloy processed by equal-channel angular pressing, Materials Science and Engineering: A 586 (2013) 108-114.

[21] J. Blaber, B. Adair, A. Antoniou, Ncorr: Open-source 2D digital image correlation Matlab software, Experimental Mechanics (2015) 1-18.

[22] C.J. Bunget, W.A. Salandro, L. Mears, Thermomechanical modeling sensitivity analysis of electrically assisted forming, Proceedings of the Institution of Mechanical Engineers, Part B: Journal of Engineering Manufacture (2013) 0954405413482304.

[23] J. Magargee, R. Fan, J. Cao, Analysis and Observations of Current Density Sensitivity and Thermally Activated Mechanical Behavior in Electrically-Assisted Deformation, Journal of Manufacturing Science and Engineering 135(6) (2013) 061022.

[24] M. Fu, W. Chan, Geometry and grain size effects on the fracture behavior of sheet metal in micro-scale plastic deformation, Materials \& Design 32(10) (2011) 4738-4746.

[25] F. Cardarelli, Materials handbook: a concise desktop reference, Springer Science \& Business Media2008.

[26] W.H. Wright, Thermal conductivity of metals and alloys at low temperatures : Part I. A survey of existing data ; Part II. The thermal conductivity of a yellow brass and of cadmium, Georgia Institute of Technology (1959).

[27] C.D. Ross, D.B. Irvin, J.T. Roth, Manufacturing aspects relating to the effects of direct current on the tensile properties of metals, Journal of Engineering Materials and Technology 129(2) (2007) 342-347.

[28] E. Hall, The deformation and ageing of mild steel: III discussion of results, Proceedings of the Physical Society. Section B 64(9) (1951) 747.

[29] Y. Li, A.J. Bushby, D.J. Dunstan, The Hall-Petch effect as a manifestation of the general size effect, Proceedings Mathematical Physical \& Engineering Sciences 472(2190) (2016).

[30] H.J. Choi, B.H. Min, J.H. Shin, D.H. Bae, Strengthening in nanostructured 2024 aluminum alloy and its composites containing carbon nanotubes, Composites Part A Applied Science \& Manufacturing 42(10) (2011) 1438-1444.

[31] T.A. Perkins, T.J. Kronenberger, J.T. Roth, Metallic forging using electrical flow as an alternative to warm/hot working, Journal of Manufacturing Science and Engineering 129(1) (2007) 84-94.

[32] W.A. Salandro, J.J. Jones, C. Bunget, L. Mears, J.T. Roth, Introduction to Electrically Assisted Forming, Electrically Assisted Forming, Springer2015, pp. 23-36. 


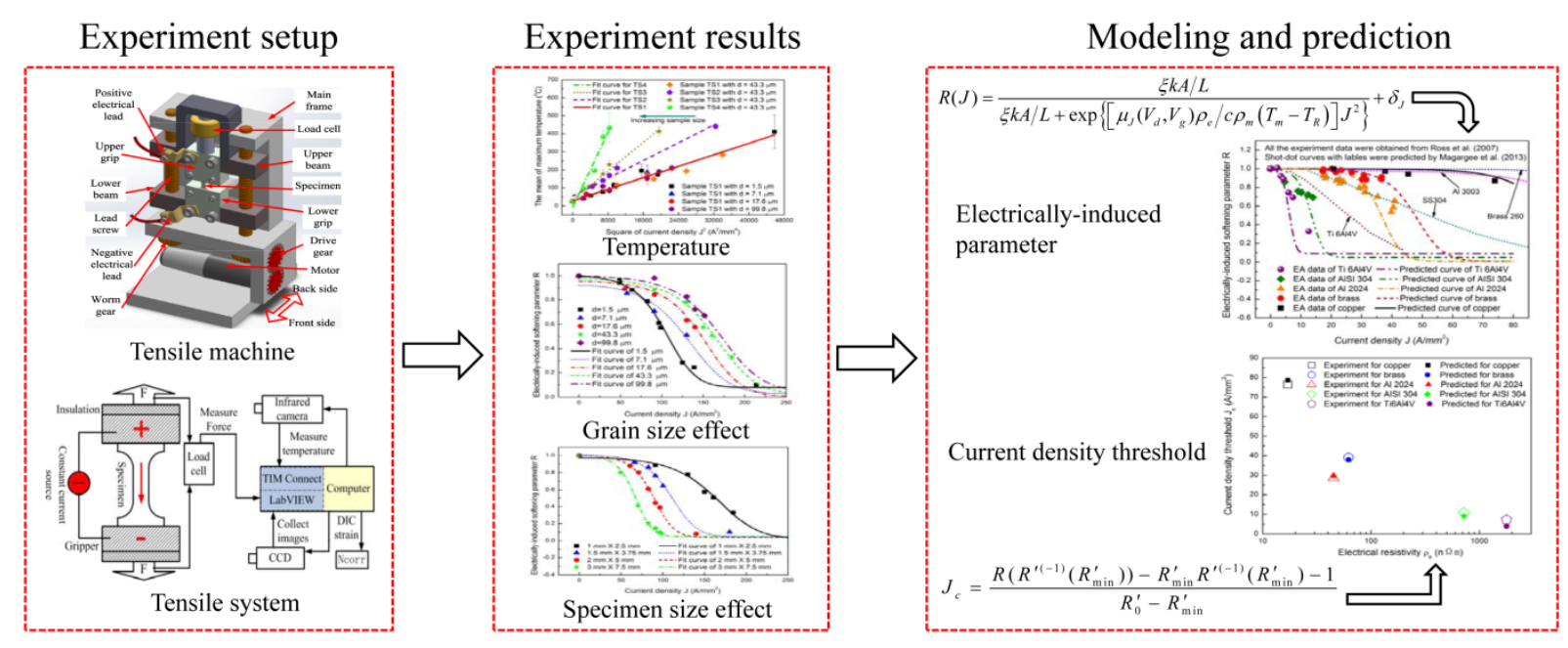

Graphical abstract 